\title{
Transcription factor 7 like 2 promotes oligodendrocyte differentiation and remyelination
}

\author{
CHAO WENG ${ }^{*}$, MAN DING ${ }^{*}$, SHANGHUA FAN, QIAN CAO and ZUNENG LU \\ Department of Neurology, Renmin Hospital of Wuhan University, Wuhan, Hubei 430060, P.R. China
}

Received May 1, 2016; Accepted March 23, 2017

DOI: $10.3892 / \mathrm{mmr} .2017 .6843$

\begin{abstract}
Transcription factor 7 like 2 (TCF7L2, also termed TCF4), is a Wnt effector induced transiently in the oligodendroglial lineage. The current well accepted hypothesis is that TCF7L2 inhibits oligodendrocyte differentiation and remyelination through canonical Wnt/ $\beta$-catenin signaling. However, recent studies indicated that TCF7L2 activity is required during oligodendrocyte differentiation and remyelination. In order to clarify this, in situ hybridization, immunofluorescence and western blot analysis using in vivo TCF7L2 conditional knockout mice, were performed and it was found that TCF7L2 promotes oligodendrocyte differentiation during myelin formation and remyelination. Furthermore, it was established that TCF7L2 does not affect oligodendrocyte precursor cells during remyelination. These data are of important clinical significance to develop novel therapeutic targets to overcome multiple sclerosis and other demyelinating diseases.
\end{abstract}

\section{Introduction}

Myelin is the spirally wrapped cell membrane that surrounds and insulates axons in the peripheral and central nervous systems (PNS and CNS, respectively). CNS myelin

Correspondence to: Dr Zuneng Lu, Department of Neurology, Renmin Hospital of Wuhan University, 238 Jiefang Road, Wuchang, Wuhan, Hubei 430060, P.R. China

E-mail: zunenglu@163.com

${ }^{*}$ Contributed equally

Abbreviations: TCF7L2, transcription factor 7 like 2; PNS, peripheral nervous system; CNS, central nervous system; OL, oligodendrocyte; OPC, oligodendrocyte precursor cells; MS, multiple sclerosis; LEF1, lymphoid enhancer binding factor 1; cKO, conditional knockout; PLP1, proteolipid protein 1; APC, adenomatous polyposis coli; CNP, 2',3'-cyclic nucleotide 3' phosphodiesterase; MBP, myelin basic protein; PDGFRA, platelet-derived growth factor receptor $\alpha$

Key words: transcription factor 7 like 2, oligodendrocyte differentiation, myelination, remyelination is synthesized by oligodendrocyte (OL). OL myelination permits salutatory propagation of nerve signals, and is critical for cognitive and motor functions in the CNS (1). Myelination is a multistep process involving the proliferation of OL precursor cells (OPCs), timely differentiation into postmitotic OLs, ensheathment of axons, initiation of myelin wrapping, and expansion of the myelin sheath during myelination (2). Independent of underlying mechanisms and causes, demyelinating diseases, such as multiple sclerosis (MS), generally result in permanent damage, functional loss and persisting disabilities. Such demyelination, however, often triggers a spontaneous myelin repair process, also termed remyelination (3). Remyelination results in myelin reconstitution and functional recovery via recruitment and activation of resident OPCs that differentiate and replace lost OLs (4). Remyelination is best investigated in models where demyelination occurs at a predictable anatomical site, and follows a well-defined and reproducible kinetic and time schedule.

A primary demyelinating disease is MS, which is a recurrent progressive disease. It is estimated that $\sim 2.5$ million people worldwide suffer from MS (5). The majority are young adults (age, 20-40 years) with females outnumbering males by $2: 1$ (5). The specific symptoms are determined by the location of the demyelinated lesions. They may include muscle weakness, loss of sensitivity, incontinency, visual troubles, ataxia, fatigue and mood alterations (6). The etiology remains unclear, and there is no effective treatment for this disease.

Studies of signaling pathways, including bone morphogenetic protein/Id, Wnt/ $\beta$-catenin and Notch/Hes signaling have been shown to negatively regulate OL differentiation $(7,8)$. TCF7L2 is one of the four members of the TCF/lymphoid enhancer binding factor 1 (LEF1) family (gene symbols, TCF7L1, TCF7L2, TCF7 and LEF1) that are essential for mediating Wnt/ $\beta$-catenin signaling in Wnt-activated cells (9). Previously, many studies indicated that TCF7L2 expression in the oligodendroglial lineage cells inhibits or delays OL differentiation, and inhibits remyelination in demyelinating diseases $(10,11)$. However, recently, two studies concluded that TCF7L2 positively regulates OL differentiation $(12,13)$. In addition, TCF7L2 affect OL differentiation via different mechanisms $(10,12,13)$.

The aim of the current study was to investigate the role of Tcf712 in myelination and remyelination, which may have 
clinical significance in the development novel therapeutic targets.

\section{Materials and methods}

Transgenic mice. The transgenic Cnp-Cre and Tcf $7 l 2^{f l f l}$ (exon11 flanked by loxP sites) mice were provided by Dr Hui Fu (Department of Anatomy, Basic Medical School of Wuhan University, Wuhan, China). Male and female mice were used in the current study. Tcf7l2 $f / f l$ and Cnp-cre. C57BL/6 served as the genetic background for the $T c f 7 l 2^{f l f l}$ mice. All animal experiments were performed in accordance with the National Institutes of Health guide for the care and use of Laboratory animals (NIH publications no. 8023, revised 1978) and were approved by Institutional Animal Care and Use Committee at Wuhan University (Wuhan, China).

$m R N A$ in situ hybridization. Sections containing the spinal cord $(25.4 \times 76.2 \mathrm{~mm})$ were hybridized overnight with a labeled RNA probe $(0.8-1.2 \mu \mathrm{g} / \mathrm{ml})$ at $65^{\circ} \mathrm{C}$. The sections were washed in $2 \mathrm{X}$ saline-sodium citrate $(\mathrm{SSC})$ at $67^{\circ} \mathrm{C}$, incubated with RNase $(1 \mu \mathrm{g} / \mathrm{ml}, 2 \mathrm{X} \mathrm{SSC})$ at $37^{\circ} \mathrm{C}$, washed in $0.2 \mathrm{X} \mathrm{SSC}$ at $67^{\circ} \mathrm{C}$, blocked in $1 \mathrm{X}$ phosphate-buffered saline (PBS) with $10 \%$ lamb sera, and incubated in alkaline phosphatase-labeled anti-digoxigenin antibody (catalog no. 11093274910; Roche Diagnostics GmbH-Mannheim, Germany; 1:2,000, 10\% lamb sera) overnight at $4^{\circ} \mathrm{C}$. Sections were washed and stained with nitro blue tetrazolium and 5-bromo-4-chloro-3-indolyl phosphate or BM purple (Roche Diagnostics $\mathrm{GmbH}$ ). Staining was terminated following visual inspection using a light microscope. Finally, sections were washed in 1X PBS three times, fixed in $4 \%$ paraformaldehyde and coverslipped with glycerol.

Immunofluorescence and antibodies. Slides were incubated with antibodies (anti-CNP, catalog no. NE1020, 1:200, Calbiochem; anti-APC, catalog no. OP80, 1:60, EMD Millipore, Billerica, MA, USA; anti-PDGFRA, catalog no. APA5, 1:300, BD Bioscience, Franklin Lakes, NJ, USA) overnight at $4^{\circ} \mathrm{C}$. Sections were then washed three times with $1 \mathrm{X}$ PBS, incubated with Alexa-488- or Alexa-555-conjugated secondary antibodies (goat anti-mouse IgG-Alexa Flour 555, catalog no. A21422, 1:500, Cell Signaling Technology, Inc., Danvers, MA, USA; Goat anti-rabbit IgG-Alexa Flour 488, catalog no. 4412s, 1:500, Cell Signaling Technology, Inc.) and fluorescent images were obtained using a Nikon epifluorescence microscope (Nikon Corporation, Tokyo, Japan). Antibodies used in the study were as follows: Anti-APC, WNT signaling pathway regulator (APC; 1:60; cat. no. OP80; EMD Millipore), anti-2',3'-cyclic nucleotide 3' phosphodiesterase (CNP; 1:200; cat. no. NE1020; EMD Millipore), anti-myelin basic protein (MBP; 1:200; cat. no. NE1018; EMD Millipore, ) and anti-platelet-derived growth factor receptor $\alpha$ (PDGFRA; 1:300; cat. no. APA5; BD Biosciences).

Protein extraction and western blotting. For western blot analysis, whole cell lysates were prepared from the corpus callosum at the thirtieth day after birth (P30). using RIPA buffer (Applygen Technologies, Inc., Beijing, China), phenylmethylsulfonyl fluoride (Biosharp, Shanghai, China) and
PhosSTOP (Roche Diagnostics GmbH). The tissue samples were minced using an electric homogenizer. The protein concentrations in the centrifugation-clarified cell lysates were measured using a bicinchoninic acid Protein Assay kit (Pierce; Thermo Fisher Scientific, Inc., Waltham, MA, USA) and equal quantities of protein were separated on $10 \%$ SDS-PAGE gel and transferred (100 V for $2 \mathrm{~h}$ ) to Hybond polyvinylidene fluoride membranes (GE Healthcare Life Sciences, Shanghai, China). For Western blotting, primary antibodies against CNP (1:100), MBP (1:1,000), GAPDH $(1: 1,000), \beta$-actin $(1: 10,000)$ were used. Signals were developed using horseradish peroxidase-conjugated secondary antibodies (goat anti-mouse antibody conjugated to horse radish peroxidase, 1:5,000, catalog no. AS1106, Aspen Biotechnology Co., Ltd., Hubei, China); goat anti-rabbit antibody conjugated to horse radish peroxidase, 1:5,000, catalog no. AS1107, Aspen Biotechnology Co., Ltd.) and an ECL kit (GE Healthcare Life Science).

Eriochrome cyanine. Slides were washed in $1 \mathrm{X}$ PBS three times, and dyed with eriochrome cyanine (catalog no. B100835-5G, Shanghai Aladdin Bio-Chem Technology Co., Ltd., Shanghai, China) for $30 \mathrm{~min}$, and then washed three times with $1 \mathrm{X}$ PBS. Sections were differentiated by $10 \%$ iron alum (catalog no. 10009218, Sinopharm Chemical Reagent Co., Ltd., Shanghai, China) for $8 \mathrm{~min}$, and washed three times with $1 \mathrm{X}$ PBS, prior to coverslipping with neutral gum (catalog no. 1000416, Sinopharm Chemical Reagent Co., Ltd.).

Rotarod performance test. The rotarod performance test is based on a rotating rod with forced motor activity applied by a mouse. The test is used to measure parameters, such as riding time (sec) or endurance. In the rotarod apparatus (IITC Life Science, Woodland Hills, CA, USA), mice were placed individually on a horizontally oriented, rotating cylinder suspended above a cage floor, which is low enough not to injure the animal, but high enough to induce avoidance of fall. The fall off time of the mouse from the rotating rod was recorded. The difference in fall off times from the rotating rod between the two groups was taken as an index of muscle relaxation and motor coordination activity.

Cuprizone test. The TCF7L2 cKO and control mice (age, 8-10 weeks) were fed with a mixture of $0.2 \%$ cuprizone (Sigma-Aldrich; Merck KGaA, Darmstadt, Germany) in ground chow for up to 6 weeks. Cuprizone is a copper chelator that induces demyelination of the corpus callosum if administered orally to adult mice. A normal diet was provided for 2 weeks after cuprizone treatment. The demyelination peaked in the fifth week. The remyelination occurred thereafter and was allowed to develop for two weeks during the current study.

Statistical analysis. Quantification was performed on data from at least three independent experiments, and the data were presented as means \pm standard error of the mean in the graphs. Student's t-test was used for comparisons between two sets of data. ImageJ version 2.0 (National Institutes of Health, Bethesda, MD, USA) was used for quantitative 


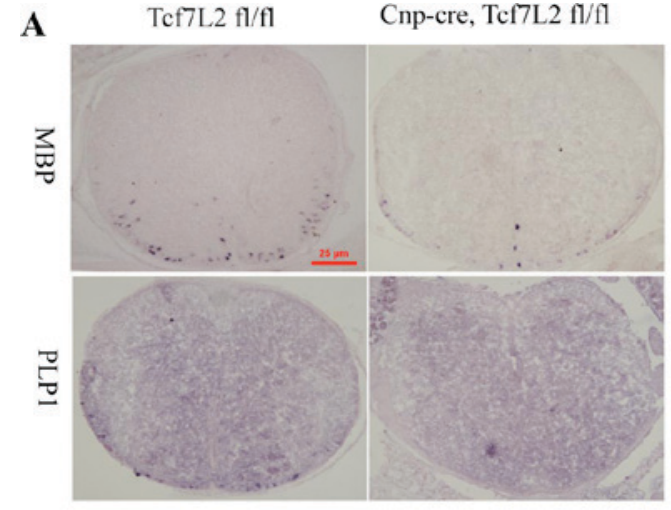

C

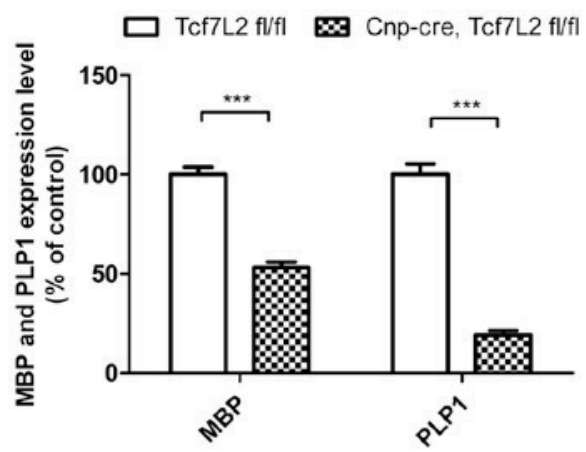

B

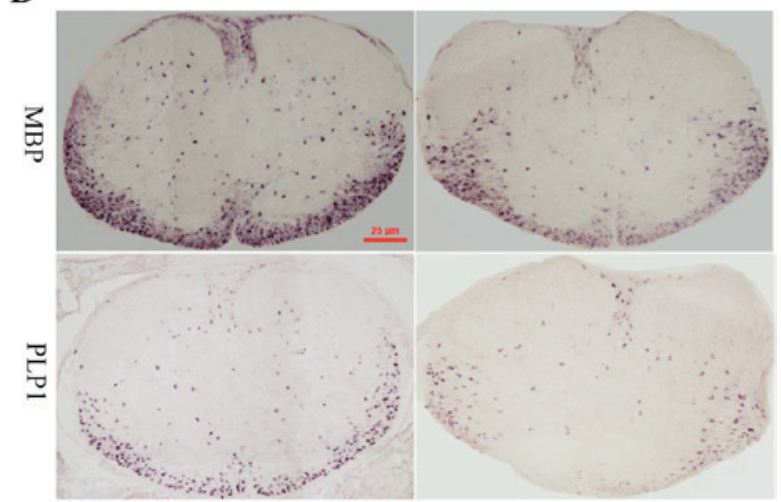

D

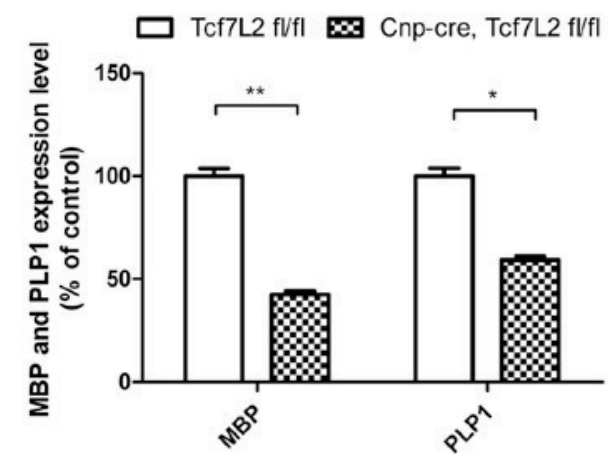

Figure 1. TCF7L2 cKO inhibits OL differentiation. (A) TCF7L2 cKO mice demonstrated that the mRNA expression level of MBP and PLP1 decreased significantly when compared with the control group in the spinal cord at P0 ( $\mathrm{n}=5$ per group). Magnification, x100. (B) The TCF7L2 cKO mice demonstrated significantly decreased expression levels of MBP and PLP1 mRNA in the spinal cord when compared with the control group at P7 (n=4 per group). Magnification, x100. (C) Quantitative analysis indicated that the mRNA expression levels of MBP and PLP1 in the TCF7L2 cKO group were significantly reduced compared with that of the control group at P0 (P<0.001). (D) Quantitative analysis further indicated that the MBP and PLP1 mRNA expression levels in the TCF7L2 cKO group were significantly lower than those of the control group at $\mathrm{P} 7$ (MBP, $\mathrm{P}<0.01 ; \mathrm{PLP}, \mathrm{P}<0.05$ ). ${ }^{*} \mathrm{P}<0.05,{ }^{* *} \mathrm{P}<0.01$ and ${ }^{* * * *} \mathrm{P}<0.001$. Error bars indicate the standard error of the mean. TCF7L2, transcription factor 7 like 2; cKO, conditional knockout; OL, oligodendrocyte; MBP, myelin basic protein; PLP1, proteolipid protein 1. P0, the first day after birth; P7, the seventh day after birth.

analysis of the mRNA and protein expression levels of MBP, proteolipid protein 1 (PLP1), CNP, APC and PDGFRA. $\mathrm{P}<0.05$ was considered to indicate statistically significant differences.

\section{Results}

TCF7L2 deletion inhibits postnatal OL differentiation during myelin formation. The Cre-loxP system was used to conditionally knock out TCF7L2 in oligodendroglial lineage cells, and avoid the lethality of TCF7L2-null newborns (14). Cnp-cre and $T c f 7 l 2^{f l f l}$ (exon11 is flanked by loxP sites) mice were used to delete the TCF7L2 exon11 sequence in the OPCs, which is the DNA binding domain (15).

Knockout of the TCF7L2 exon 11 sequence in the oligodendroglial lineage cells (Fig. 1) demonstrated that the mRNA levels of MBP and PLP1 in the spinal cords were significantly lower when compared with those of the control group at $\mathrm{P} 0(\mathrm{P}<0.001$; Fig. $1 \mathrm{~A}$ and $\mathrm{C})$, and a similar reduction in the spinal cords was observed at $\mathrm{P} 7$ (MBP, $\mathrm{P}<0.01$; $\mathrm{PLP}, \mathrm{P}<0.05$; Fig. 1B and D) indicating that TCF7L2 positively regulates OL differentiation. Furthermore, corpus callosum samples were obtained, and protein samples from the Cnp-cre-mediated TCF7L2 KO mice and littermate control mice were extracted at P30. Western blot analysis revealed markedly decreased expression levels of CNP and MBP in the TCF7L2 cKOs compared with the controls (Fig. 2A and B). Quantitative analysis demonstrated that the expression levels of CNP and MBP were significantly lower than those of the control mice $(\mathrm{P}<0.01$; Fig. 2C). In order to exam motor coordination activity, the rotarod performance test demonstrated that the riding time on the rotating rod was significantly shorter in the TCF7L2 cKO group when compared with the control mice at P30, P45 and P60 ( $\mathrm{P}<0.05$; Fig. 3). The riding time of the TCF7L2 cKO group mice was decreasing at P90 and P120 $(\mathrm{P}=0.131$ and $\mathrm{P}=0.087$, respectively). Together, these data indicate that TCF7L2 promotes OL differentiation and myelination during developmental myelination.

TCF7L2 cKO inhibits OL differentiation during remyelination. Various previous studies have proposed that TCF7L2 may inhibit OL differentiation and oligodendrogenesis during myelin formation $(16,17)$. There are few studies that have revealed the role of TCF7L2 in remyelination. Based on the current data, it was hypothesized that TCF7L2 promotes OL differentiation during remyelination. Among the various animal and cell models available to investigate remyelination, the cuprizone model stands out due to its reproducibility, simplicity to induce demyelination and low mortality rates (18). In the present study, the $0.2 \%$ cuprizone demyelination/remyelination model was used to determine the role of Tcf712 in remyelination. 


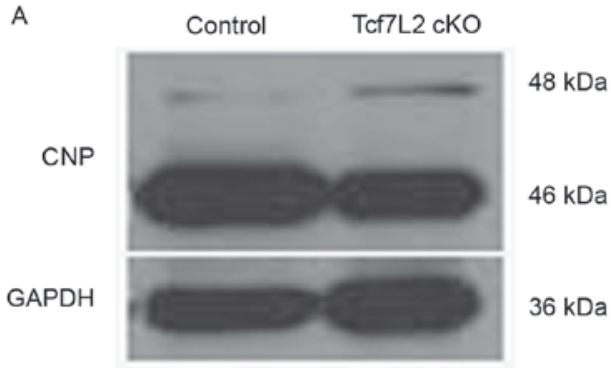

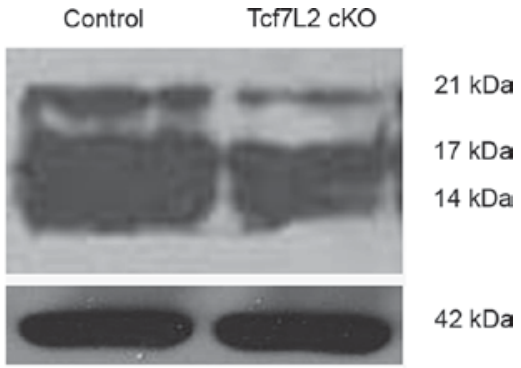

C

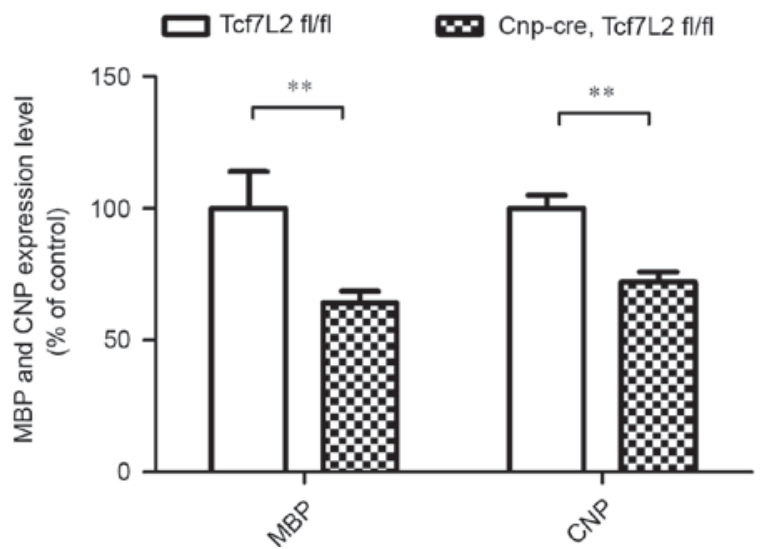

Figure 2. Expression levels of CNP and MBP proteins were reduced in the TCF7L2 cKO group. (A) Western blot analysis of the P30 corpus callosum homogenates (10 $\mu \mathrm{g}$ total protein per lane) exhibited significantly reduced expression levels of CNP (46 kDa) in the TCF7L2 cKO mice compared with the littermate controls ( $\mathrm{n}=3$ per group). (B) Western blot analysis indicated a significant decrease in the expression level of MBP (14, 17 and $21 \mathrm{kDa}$ ) in the TCF7L2 cKO mice compared with the littermate controls ( $\mathrm{n}=3$ per group). GAPDH (36 kDa) and $\beta$-actin (42 kDa) served as loading controls. (C) Quantitative analysis of western blot further indicated that the expression levels of CNP (46 and $48 \mathrm{kDa}$ ) and MBP (14, 17 and $21 \mathrm{kDa})$ were significantly lower than those of the controls. Error bars indicate standard error of the mean. ${ }^{* *} \mathrm{P}<0.01$. CNP, 2', 3'-cyclic nucleotide 3 ' phosphodiesterase; MBP, myelin basic protein; TCF7L2, transcription factor 7 like 2; cKO, conditional knockout; P30, the thirtieth day after birth.

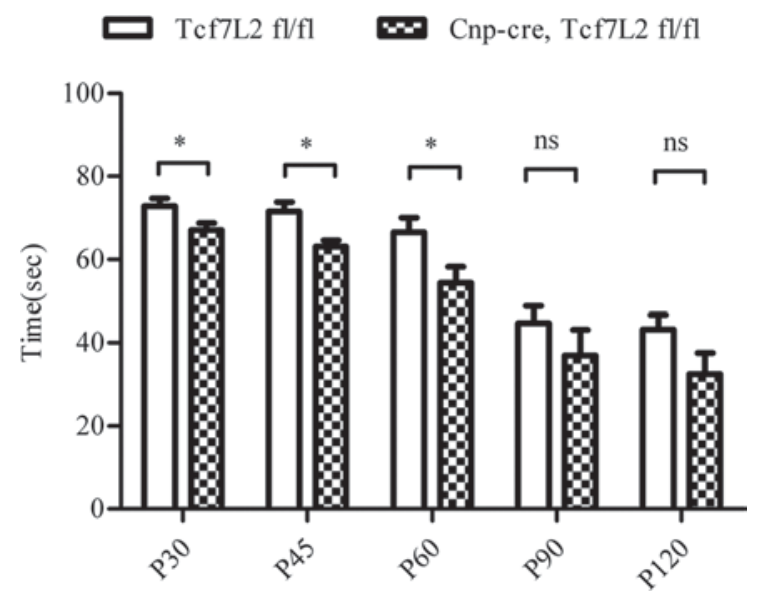

Figure 3. Behavioral experiments from TCF7L2 cKO and littermate control mice were analyzed. The rotarod performance tests demonstrated that the riding times of the TCF7L2 cKO mice on the rotarod instrument were significantly shorter than the controls at $\mathrm{P} 30, \mathrm{P} 45$ and $\mathrm{P} 60(\mathrm{P}<0.05)$. The riding time of the mice in the TCF7L2 cKO group decreased at P90 and $\mathrm{P} 120(\mathrm{P}=0.131$ and $\mathrm{P}=0.087$, respectively). $\mathrm{n}=9$ per group. Error bars indicate standard error of the mean. ${ }^{*} \mathrm{P}<0.05$. TCF7L2, transcription factor 7 like 2 ; cKO, conditional knockout; CNP, 2',3'-cyclic nucleotide 3' phosphodiesterase; ns, not significant.

After feeding mice $0.2 \%$ cuprizone for 6 weeks, myelin histochemistry with Eriochrome cyanine revealed that there was almost no myelin in the corpus callosum samples between the two groups (Fig. 4A). The magnified view demonstrated further that the corpus callosum samples were fully demyelinated in the two groups (Fig. 4A).

After returning to normal chow feed for 2 weeks, the corpus callosum samples were almost completely remyelinated in the control mice (Fig. 4B and C). By contrast, remyelination did not occur in the TCF7L2 cKO mice (Fig. 4B and C). Furthermore, immunofluorescence demonstrated that the expression levels of APC and CNP in the control group were significantly higher than the TCF7L2 cKO group (Fig. 5A and $\mathrm{B}$ ) and almost the same as normal expression levels of APC and CNP (data not shown). Quantitative analysis further indicated that the expression levels of APC and CNP were significantly lower than those of the control mice $(\mathrm{P}<0.001$; Fig. 5C). In addition, the mRNA expression level of MBP observed by in situ hybridization was significantly higher in the control group (data not shown). These data indicate that TCF7L2 cKO inhibits remyelination, whereas it promotes OL differentiation during remyelination.

TCF7L2 does not affect OPCs during remyelination. To determine whether OPCs were perturbed in TCF7L2 cKO mice, the expression level of PDGFRA, an OL precursor cell marker, was examined during remyelination. After returning to feeding with normal chow for 2 weeks, the 
A

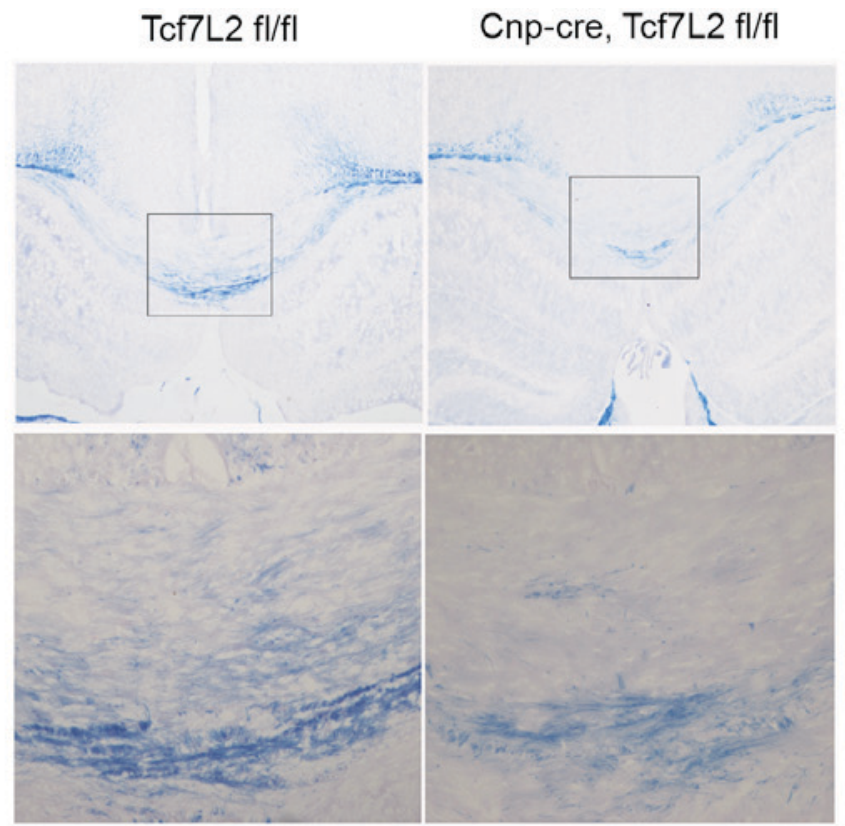

B Tcf7L2 fl/fl

Cnp-cre, Tcf7L2 fl/fl

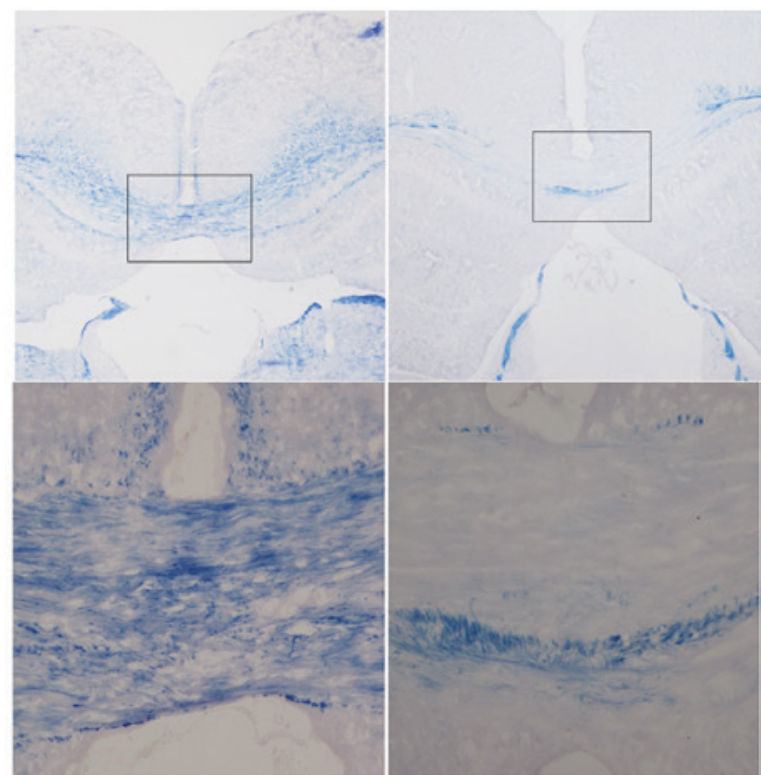

Figure 4. TCF7L2 cKO inhibits remyelination. (A) Subsequent to feeding the mice $0.2 \%$ cuprizone for 6 weeks, myelin histochemistry with eriochrome cyanine demonstrated that there was almost no myelin in the corpus callosum samples between the two groups $(\mathrm{n}=4$ per group; magnification, $\mathrm{x} 100)$. The bottom row presents the magnified images of the boxed areas in the top row (magnification, x200). (B) Subsequent to returning to feeding with normal chow for 2 weeks, the corpus callosum samples were almost completely remyelinated in the control group ( $\mathrm{n}=5$ per group; magnification, $\mathrm{x} 100)$. By contrast, remyelination did not occur in the TCF7L2 cKO mice. The bottom row presents the magnified images of the boxed areas in the top row (magnification, $\mathrm{x} 200$ ). TCF7L2, transcription factor 7 like 2; cKO, conditional knockout; CNP, 2',3'-cyclic nucleotide 3' phosphodiesterase.

Tcf7L2 fl/fl

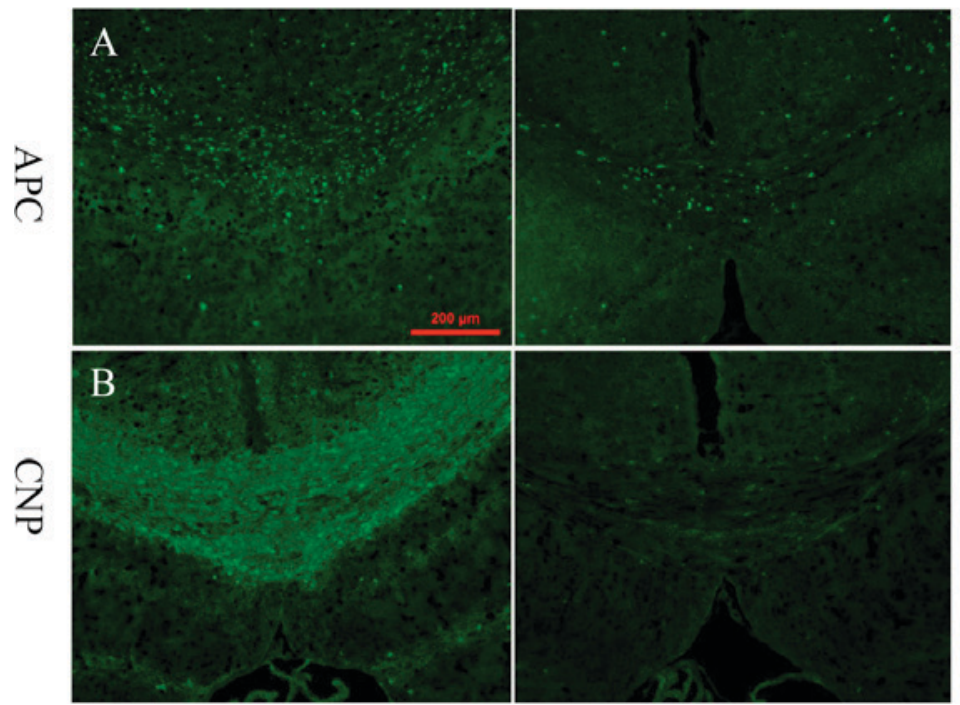

Cnp-cre, Tcf7L2 fl/fl

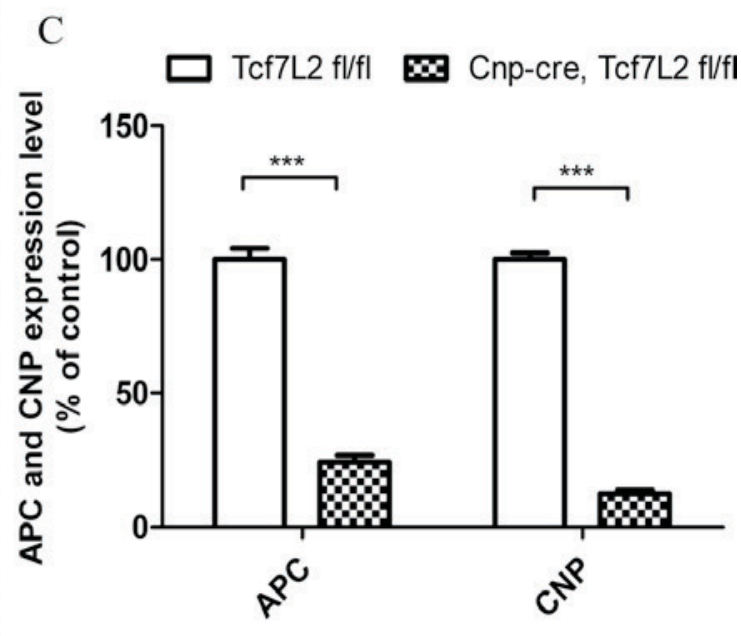

Figure 5. TCF7L2 promotes remyelination. After returning to feeding with normal chow for 2 weeks, immunofluorescence indicated that the expression levels of (A) APC and (B) CNP in the control mice were significantly higher than in the TCF7L2 cKO mice (n=5 per group). (C) Quantitative analysis further indicated that the expression levels of APC and CNP in the TCF7L2 cKO mice were significantly less than those of the control mice (P<0.001; $\mathrm{n}=5$ per group). ${ }^{* * * *} \mathrm{P}<0.001$. Error bars indicate the standard error of the mean. Magnification, $\mathrm{x} 100$. TCF7L2, transcription factor 7 like 2; cKO, conditional knockout; APC, adenomatous polyposis coli; CNP, 2',3'-cyclic nucleotide 3' phosphodiesterase.

expression level of PDGFRA was almost the same in the two groups during remyelination (Fig. 6Aa and $\mathrm{Ab}$ ). Quantitative analysis further indicated that there was no significant alteration in terms of OPCs between the two groups during remyelination $(\mathrm{P}>0.05$; Fig. $6 \mathrm{~B})$. These data indicate that TCF7L2 cKO does not affect OPCs during remyelination.

\section{Discussion}

There were various significant findings in the present study. In contrast to previous reports, TCF7L2 promoted postnatal OL differentiation during myelination. In addition, TCF7L2 positively regulated OL differentiation during remyelination and finally, TCF7L2 did not affect OPCs during remyelination. 
A

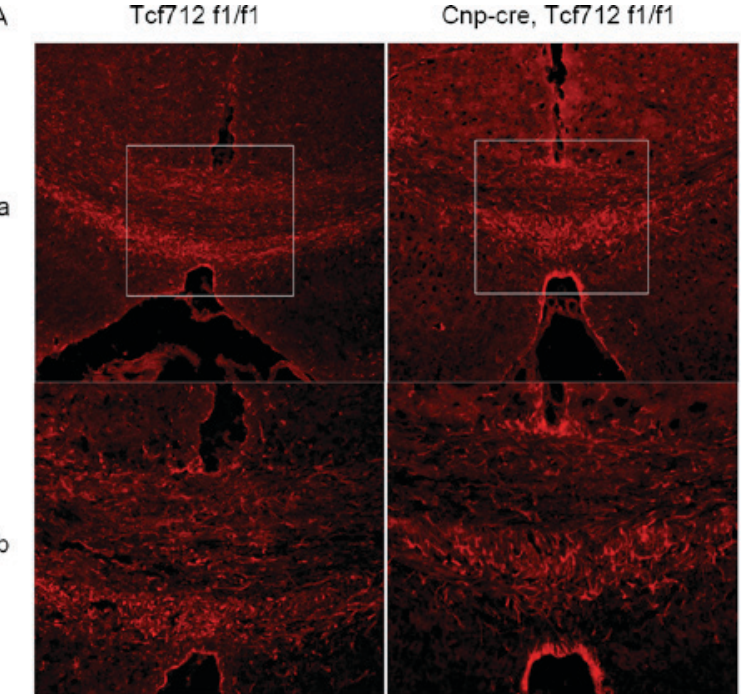
B

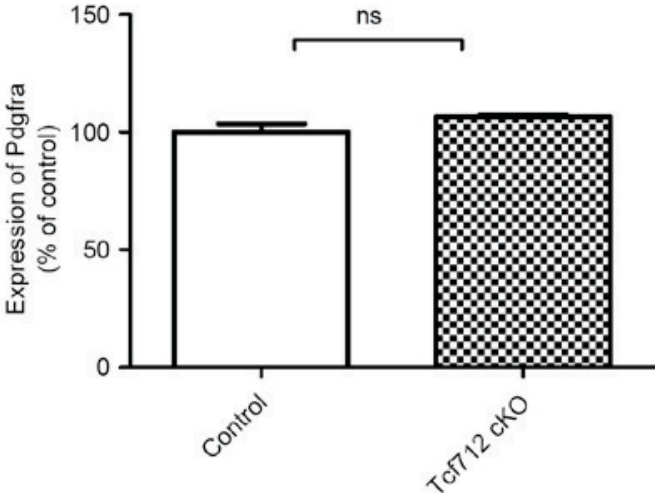

Figure 6. TCF7L2 cKO does not affect OPCs during remyelination. (Aa) After returning to feeding with normal chow for 2 weeks, immunofluorescence demonstrated that the expression of PDGFRA was almost the same in the two groups ( $\mathrm{n}=5$ per group; magnification, $\mathrm{x} 100$ ). (Ab) Magnified images of the boxed areas in the Aa (magnification, x200). (B) No significant difference was demonstrated by quantitative analysis in the expression level of PDGFRA between the two groups during remyelination (P>0.05). Error bars indicate SEM. TCF7L2, transcription factor 7 like 2; cKO, conditional knockout; CNP, 2',3'-cyclic nucleotide $3^{\prime}$ phosphodiesterase; PDGFRA, platelet-derived growth factor receptor $\alpha$. ns, not significant.

It is well accepted that TCF7L2 is one of the four members of the TCF/LEF1 family (TCF7, TCF711, TCF712 and LEF1), which are essential for mediating canonical Wnt/ $\beta$-catenin signaling in Wnt-activated cells (9). Previous studies indicated that TCF7L2 inhibits OL differentiation $(16,17)$, while a recent study reported that TCF7L2 promotes OL differentiation (13). According to existing studies, it is unclear whether TCF7L2 inhibits or promotes OL differentiation. The results presented here do not support the hypothesis that TCF7L2 inhibits OL differentiation and myelination via Wnt/ $\beta$-catenin activation. The current study focused on the neonatal and postnatal oligodendroglial lineage and demonstrated that, contrary to the well accepted hypothesis that TCF7L2 inhibits OL differentiation and myelination via Wnt/ $\beta$-catenin activation, TCF7L2 functions as a positive regulator of OL differentiation and myelination during normal developmental myelination. This conclusion is consistent with previous data derived from TCF7L2-null late embryos or newborns $(13,19)$.

In addition, the cuprizone demyelination/remyelination model was used to determine the role of TCF7L2 in remyelination. Various studies have provided compelling evidence of the usefulness of this model to discover novel therapeutic options to boost remyelination $(20,21)$. Remyelination, which resembles the physiological process of myelination and has a high degree of complexity and regulation, is classified into four consecutive steps: Proliferation of OPCs, migration of progenitor cells towards the demyelinated axons, OPC differentiation and interaction of premature OLs with the denuded axon (3). These steps are regulated by many intrinsic and extrinsic factors. For example, transcription factors, Sonic hedgehog and oligodendrocyte transcription factor 1 have been identified as important intrinsic regulators for OL differentiation and myelination $(22,23)$, whereas astrocytes are regarded as important extrinsic regulators for myelin repair. In the present study, immunohistochemistry was used against the major myelin proteins, APC and CNP, and myelin histochemistry with eriochrome cyanine was used to quantify remyelination between the two groups. It was demonstrated that TCF7L2 promotes remyelination, which is indispensable for clinical demyelinating diseases. The conclusion concerning the role of TCF7L2 in remyelination is compatible with a recent study (13).

The ablation of TCF7L2 directed by Cnp-cre does not affect OPCs during remyelination. This demonstration most likely reflects a specific requirement of TCF7L2 in OL differentiation and remyelination rather than a general requirement in OPCs.

While many regulators of OL differentiation and myelination have been identified, the specific mechanisms remain poorly understood. As TCF7L2 is an essential effector for Wnt/ $\beta$-catenin signaling, various previous studies indicated that TCF7L2 inhibits oligodendrogenesis and OL differentiation through $\mathrm{Wnt} / \beta$-catenin activation $(17,24)$. One study concluded that TCF7L2 positively regulates OL differentiation independent of $\mathrm{Wnt} / \beta$-catenin signaling. Whereas a recent study demonstrated that TCF7L2 interacts with a transcriptional co-repressor, Kaiso to block $\beta$-catenin signaling at the differentiation onset, and subsequently TCF7L2 recruits and cooperates with SRY-box 10 to promote myelination during OL maturation (12).

In conclusion, TCF7L2/TCF4 is required for OL differentiation and remyelination; however, the mechanism by which TCF7L2 controls myelination and remyelination remains unknown. Additional studies are required to elucidate how TCF7L2 promotes OL differentiation and remyelination, which is of particular clinical importance in the diagnosis and treatment of demyelinating diseases.

\section{Acknowledgements}

The authors would like to thank Dr Hui Fu (Department of Anatomy, Basic Medical School of Wuhan University, Wuhan, 
China) for providing the Cnp-cre and $T c f 7 l 2^{f l f l}$ mice. The study was supported by the Fundamental Research Funds for the Central Universities of China (2042017kf0129), grants from the Health and Family Planning Commission of Hubei Province Scientific Research Project (WJ2015MA007) and Wuhan Science and Technology Bureau Scientific Research Project (2015060101010047).

\section{References}

1. Bercury KK and Macklin WB: Dynamics and mechanisms of CNS myelination. Dev Cell 32: 447-458, 2015.

2. Ishii A, Fyffe-Maricich SL, Furusho M, Miller RH and Bansal R: ERK1/ERK2 MAPK signaling is required to increase myelin thickness independent of oligodendrocyte differentiation and initiation of myelination. J Neurosci 32: 8855-8864, 2012.

3. Franklin RJ and Ffrench-Constant C: Remyelination in the CNS: From biology to therapy. Nat Rev Neurosci 9: 839-855, 2008.

4. El Waly B, Macchi M, Cayre M and Durbec P: Oligodendrogenesis in the normal and pathological central nervous system. Front Neurosci 8: 145, 2014.

5. Milo R and Kahana E: Multiple sclerosis: Geoepidemiology, genetics and the environment. Autoimmun Rev 9: A387-A394, 2010.

6. Compston A and Coles A: Multiple sclerosis. Lancet 372: $1502-1517,2008$.

7. Zuchero JB and Barres BA: Intrinsic and extrinsic control of oligodendrocyte development. Curr Opin Neurobiol 23: 914-920, 2013.

8. He L and Lu QR: Coordinated control of oligodendrocyte development by extrinsic and intrinsic signaling cues. Neurosci Bull 29: 129-143, 2013.

9. Fancy SP, Kotter MR, Harrington EP, Huang JK, Zhao C, Rowitch DH and Franklin RJ: Overcoming remyelination failure in multiple sclerosis and other myelin disorders. Exp Neurol 225: 18-23, 2010.

10. Lürbke A, Hagemeier K, Cui QL, Metz I, Brück W, Antel J and Kuhlmann T: Limited TCF7L2 expression in MS lesions. PLoS One 8: e72822, 2013.

11. Fancy SP, Harrington EP, Yuen TJ, Silbereis JC, Zhao C, Baranzini SE, Bruce CC, Otero JJ, Huang EJ, Nusse R, et al: Axin2 as regulatory and therapeutic target in newborn brain injury and remyelination. Nat Neurosci 14: 1009-1016, 2011.

12. Zhao C, Deng Y, Liu L, Yu K, Zhang L, Wang H, He X, Wang J, $\mathrm{Lu} \mathrm{C}, \mathrm{Wu} \mathrm{LN}$, et al: Dual regulatory switch through interactions of Tcf712/Tcf4 with stage-specific partners propels oligodendroglial maturation. Nat Commun 7: 10883, 2016.
13. Hammond E, Lang J, Maeda Y, Pleasure D, Angus-Hill M, Xu J, Horiuchi M, Deng W and Guo F: The Wnt effector transcription factor 7-like 2 positively regulates oligodendrocyte differentiation in a manner independent of $\mathrm{Wnt} / \beta$-catenin signaling. J Neurosci 35: 5007-5022, 2015.

14. Boj SF, van Es JH, Huch M, Li VS, José A, Hatzis P, Mokry M, Haegebarth A, van den Born M, Chambon P, et al: Diabetes risk gene and Wnt effector Tcf712/TCF4 controls hepatic response to perinatal and adult metabolic demand. Cell 151: 1595-1607, 2012.

15. van Es JH, Haegebarth A, Kujala P, Itzkovitz S, Koo BK, Boj SF Korving J, van den Born M, van Oudenaarden A, Robine $S$ and Clevers H: A critical role for the Wnt effector Tcf4 in adult intestinal homeostatic self-renewal. Mol Cell Biol 32: 1918-1927, 2012.

16. Ye F, Chen Y, Hoang T, Montgomery RL, Zhao XH, Bu H, Hu T, Taketo MM, van Es JH, Clevers H, et al: HDAC1 and HDAC2 regulate oligodendrocyte differentiation by disrupting the beta-catenin-TCF interaction. Nat Neurosci 12: 829-838, 2009.

17. Wood TL, Bercury KK, Cifelli SE, Mursch LE, Min J, Dai J and Macklin WB: mTOR: A link from the extracellular milieu to transcriptional regulation of oligodendrocyte development. ASN Neuro 5: e00108, 2013.

18. Zendedel A, Beyer C and Kipp M: Cuprizone-induced demyelination as a tool to study remyelination and axonal protection. J Mol Neurosci 51: 567-572, 2013.

19. Fu H, Cai J, Clevers H, Fast E, Gray S, Greenberg R, Jain MK, Ma Q, Qiu M, Rowitch DH, et al: A genome-wide screen for spatially restricted expression patterns identifies transcription factors that regulate glial development. J Neurosci 29: 11399-11408, 2009.

20. VonDran MW, Singh H, Honeywell JZ and Dreyfus CF: Levels of BDNF impact oligodendrocyte lineage cells following a cuprizone lesion. J Neurosci 31: 14182-14190, 2011.

21. Hussain R, Ghoumari AM, Bielecki B, Steibel J, Boehm N, Liere P, Macklin WB, Kumar N, Habert R, Mhaouty-Kodja S, et al: The neural androgen receptor: A therapeutic target for myelin repair in chronic demyelination. Brain 136: 132-146, 2013.

22. Ferent J, Zimmer C, Durbec P, Ruat M and Traiffort E: Sonic Hedgehog signaling is a positive oligodendrocyte regulator during demyelination. J Neurosci 33: 1759-1772, 2013.

23. Niu J, Mei F, Wang L, Liu S, Tian Y, Mo W, Li H, Lu QR and Xiao L: Phosphorylated olig1 localizes to the cytosol of oligodendrocytes and promotes membrane expansion and maturation. Glia 60: 1427-1436, 2012.

24. Sabo JK and Cate HS: Signalling pathways that inhibit the capacity of precursor cells for myelin repair. Int J Mol Sci 14: 1031-1049, 2013. 